\title{
Quantum Inelastic Conductance through Molecular Wires
}

\author{
H. Ness and A. J. Fisher \\ Department of Physics and Astronomy, University College London, Gower Street, London WC1E 6BT, United Kingdom
}

(Received 5 March 1999)

\begin{abstract}
We calculate nonperturbatively the inelastic effects on the conductance through a conjugated molecular-wire-metal heterojunction, including realistic electron-phonon coupling. We show that at subband-gap energies the current is dominated by quantum coherent transport of virtual polarons through the molecule. In this regime, the tunneling current is strongly increased relative to the case of elastic scattering. It is essential to describe the full quantum coherence of the polaron formation and transport in order to obtain correct physics. Our results are generally applicable to one-dimensional atomic or molecular wires.
\end{abstract}

PACS numbers: 85.30.Vw, 73.40.Gk, 73.50.-h, 73.61.Ph

Truly one-dimensional conducting structures, where the confinement lengths for electrons in two directions are of the order of atomic diameters, are currently the subject of much experimental work. Examples include fullerene nanotubes [1], organic molecules bonded to metallic electrodes [2], and dangling-bond (DB) wires created by scanning tunneling microscope lithography of the $\mathrm{H}$-saturated $\mathrm{Si}(001)$ surface $[3,4]$. The conductance properties of such structures are of great importance when one considers their potential as atomic or molecular scale electronic devices. There have been many recent theoretical investigations of electron transport in these systems: for example, Joachim et al. studied Xe atom "wires" [5] and organic molecules [6] using the "elastic scattering quantum chemistry" approach; idealized one-dimensional atomic wires sandwiched between jellium electrodes have been studied using density functional theory [7] and a recursion transfer matrix method [8], while Datta and collaborators studied organic molecules on metal electrodes [9].

All the above theoretical work was done within the approximation of elastic transport, where the electronphonon ( $e$-ph) coupling plays no role. But there are good reasons to doubt the validity of this approximation in one-dimensional atomic or molecular scale systems. In small systems, the coupling between electron and other excitations is enhanced. Furthermore, a one-dimensional metal is generally unstable towards a Peierls distortion [10]. Once such a distortion has occurred and produced a band gap, charges added into the system tend to selflocalize and cause distortions of the system which lower the band gap. Such polaronic phenomena have been studied in conducting polymers for decades [11]; electron transport usually proceeds via tunneling into polaron states arising from lattice fluctuations [12].

Motivated by this physics and the possibility of measuring the transport through one-dimensional atomic and molecular wires, we report in this Letter the first calculations of the inelastic electronic transport through an atomic-scale wire including realistic $e$-ph coupling. In our calculations, the quantum coherence of the states is fully retained, i.e., no adiabatic separation between the electronic and phonon degrees of freedom is made. We have chosen to study a conjugated molecular chain as an example since the $e$-ph coupling in such materials is relatively well understood. However the same physical principles (and therefore the qualitative features of our results) are widely applicable to all one-dimensional atomic and molecular wires.

For energies within the gap, the coherent transport is dominated by tunneling. Here we study the equivalent of the polaronic phenomena in conducting polymers for virtual (i.e., tunneling) electron injection. This has previously been included in very few transport calculations: the elastic conductivity was found for a conjugated molecule containing static solitons [13] and the classical response of a conjugated oligomer to an injected electron wave packet has been considered [14]. However, the quantum coherence between the electron and the lattice was not retained. Such coherence is kept in our approach since it is essential to treat the tunneling of objects such as polarons or solitons.

Our approach for studying the coupled $e$-ph system is inspired by the Su-Schrieffer-Heeger (SSH) Hamiltonian [11], in which a tight-binding treatment for the $\pi$ electrons is combined with a classical ball-and-spring model for a one-dimensional atomic $(\mathrm{CH})_{x}$ chain. In our model, the phonons are treated on a quantum level, the $e$-ph coupling is linear, and the electronic part is expressed in terms of the one-electron eigenstates, labeled by $n$. The Hamiltonian is

$$
\begin{aligned}
H= & \sum_{n} \epsilon_{n} c_{n}^{\dagger} c_{n}+\sum_{q} \omega_{q} a_{q}^{\dagger} a_{q} \\
& +\sum_{q, n, m} \gamma_{q n m}\left(a_{q}^{\dagger}+a_{q}\right) c_{n}^{\dagger} c_{m},
\end{aligned}
$$

where $c_{n}^{\dagger}\left(c_{n}\right)$ creates (annihilates) an electron in the $n$th electronic state with energy $\epsilon_{n}$ and $a_{q}^{\dagger}\left(a_{q}\right)$ creates (annihilates) a phonon in mode $q$. This is a general Hamiltonian, describing coupled $e$-ph systems going beyond the Holstein model. 
In this work, the electronic energies $\epsilon_{n}$, the phonon frequencies $\omega_{q}$, and the coupling matrix elements $\gamma_{q n m}$ are obtained from the SSH Hamiltonian for an isolated molecular chain [15]. The one-electron eigenstates and energies $\epsilon_{n}$ are calculated self-consistently with the atomic distortions for a given number of atoms $N_{\mathrm{a}}$ and $\pi$ electrons $N_{\mathrm{e}}$. The ground state of the neutral $\left(N_{\mathrm{e}}=N_{\mathrm{a}}\right)$ dimerized chain is our reference system, from which the phonon modes and frequencies are obtained by perturbation theory [16]. The energy change, taken to second order in the atomic displacements $x_{i}$, gives the dynamical matrix from which phonon eigenmodes $V_{q}(i)$ and frequencies are obtained. We have checked that this second-order expansion of the elastic energy is sufficient to describe the formation of a static polaron in the chain. The $e$-ph coupling matrix elements are calculated from the variation of the electronic Hamiltonian due to phonon displacements. They are transformed from a real-space representation into the electron eigenstate representation to get the $\gamma_{q n m}$ elements in Eq. (1).

We choose the eigenstate representation because we wish to consider the action of $H$ on the $\left(N_{\mathrm{a}}+1\right)$-electron [or $\left(N_{\mathrm{a}}-1\right)$-electron] Hilbert space obtained by adding (or removing) one electron to (or from) the ground state of the neutral chain. It is most straightforward to project out the addition of an electron (or hole) to the already occupied (or empty) states if we work entirely with the eigenstates. Then the $n, m$ sums in Eq. (1) run over the occupied valence band states (for hole transport) or over the unoccupied conduction band states (for electron transport). We note that it is essential to keep the $N_{\mathrm{a}}$ electrons of the reference system implicitly in the calculation since they drive the Peierls distortion in the system. We justify the inclusion of only $\left(N_{\mathrm{a}} \pm 1\right)$-electron states in the transport by noting that the mean time between electron (or hole) passages $\left(\approx 10^{-7} \mathrm{~s}\right.$ for a current of $\left.1 \mathrm{pA}\right)$ is much longer than a typical residence time in the molecule $\left(\approx 10^{-15} \mathrm{~s}\right.$ for a molecule strongly coupled to the electrodes [17]). Furthermore, we have verified that the static lattice distortions due to charging are well reproduced by considering only one half of the electronic spectrum (Fig. 1).

The degree of dimerization is conveniently represented by the staggered difference $d_{i}$ between adjacent bond lengths. Figure 1 shows the $d_{i}$ pattern induced when one additional electron is injected into the neutral isolated chain. As expected, we find a reduction of dimerization, corresponding to a polaronlike defect in the middle of the chain. For short chains $\left(N_{\mathrm{a}}=40\right.$, for example), the difference of the dimerization calculated with (a) the full electronic spectrum and an exact classical treatment of the lattice ( $\mathrm{SSH}$ model), and (b) half of the electronic spectrum and a harmonic treatment of the lattice does not exceed 7\% [18]. We also checked that only the longest wavelength optical phonon modes [19] contribute to the polaron distortion.

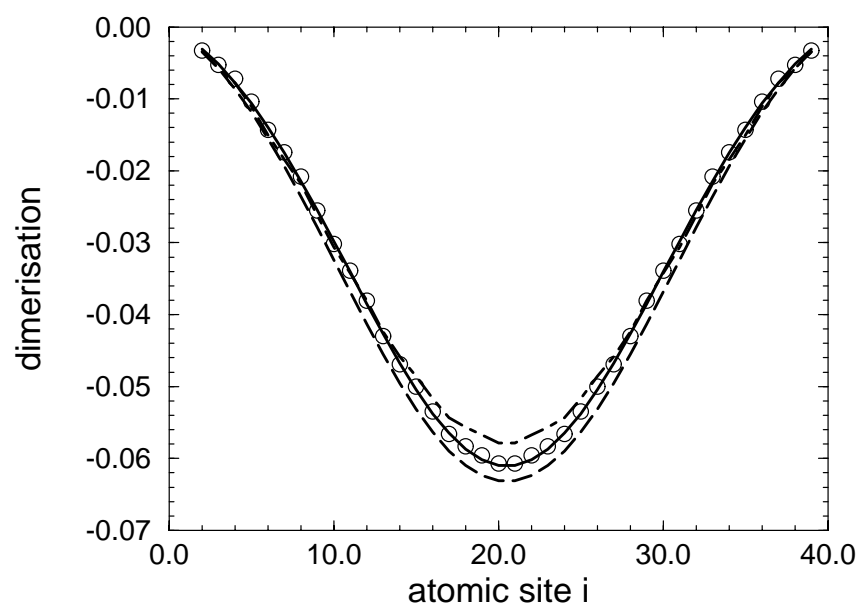

FIG. 1. Dimerization pattern $d_{i}=(-1)^{i}\left(x_{i+1}-2 x_{i}+x_{i-1}\right)$ (in $\AA$ ) induced by an additional electron in the isolated wire $\left(N_{\mathrm{a}}=40 ; N_{\mathrm{e}}=41\right)$ : classical SSH model (dashed line), half electronic spectrum and all classical phonons (solid line), $N_{\text {ph }}=6$ (lowest optical modes) classical phonons (circles), and $N_{\mathrm{ph}}=6$ (same modes) quantum phonons with $n_{\mathrm{occ}}^{\max }=6$ (dotdashed line).

The basis states for the Hamiltonian equation (1) are expressed as $\left|n,\left\{n_{q}\right\}\right\rangle$ where $n$ indexes the one-electron eigenstates and $\left\{n_{q}\right\}$ represents the set of occupation numbers for the different phonon modes $q$. The elastic states $|n,\{0\}\rangle$ have no excited phonon modes and the inelastic states $\left|n,\left\{n_{q}\right\}\right\rangle$ have phonon modes $q$ excited with $n_{q}=$ $1,2,3, \ldots, n_{\mathrm{occ}}^{\max }$. In all the calculations, the dynamical (i.e., energy dependent) correlation between the electron and the phonons is fully kept. Even for a relatively short wire the size of the complete basis set quickly becomes intractable for reasonable numerical calculations. However using a limited but sufficient number of phonon modes to describe the polaron formation permits us to treat a range of chain lengths with reasonable computing times. Furthermore, our results show that only a few excitations in each phonon mode are needed to give converged results.

Knowing $\epsilon_{n}, \omega_{q}$, and $\gamma_{q n m}$ for a realistic description of the wire, we then calculate the transport properties through the electrode/wire/electrode junction. The conjugated chain is connected to two semi-infinite 1D metallic electrodes of bandwidth $6 \mathrm{eV}$ via the use of embedding potentials $\Sigma_{L}$ and $\Sigma_{R}$ [20]. The propagation of an electron or hole through the junction is treated as a scattering problem with many channels [21-23]. Full scattering boundary conditions are applied for an incident electron or hole plane wave with the wire in a given vibrational state. For the results given in this paper, the chain configuration before scattering is the ground state (no phonons present). The solution of the scattering problem can be obtained by solving the following linear system:

$$
\left[E-H-\Sigma_{L}(E)-\Sigma_{R}(E)\right]|\alpha(E)\rangle=|s(E)\rangle,
$$

where $|s(E)\rangle$ is the source term, representing the injected carrier at energy $E$. The ket $|\alpha(E)\rangle$ is the wave function 
of the scattering state; it is represented as an expansion over the basis set $\left|n,\left\{n_{q}\right\}\right\rangle$. We can derive the transmission and reflection coefficients for the different channels, the currents, and the average atomic displacements from the solution [22,23] of Eq. (2) obtained by a conjugate gradient technique.

To check the validity of our quantum approach, we first calculate the mean atomic displacements $\left\langle x_{i}\right\rangle$ induced by adding an electron to an isolated chain in the ground state [24]. The corresponding dimerization pattern, also shown in Fig. 1, reproduces the results obtained with classical phonons. The dip in the dimerization is slightly shallower in the fully quantum case; this may result from quantum delocalization of the polaron state.

We now turn to calculations on a current-carrying wire between two electrodes. Figure 2 shows the differential conductance $\sigma(E)$ of the junction. Calculations are performed with different numbers of phonon modes and with different maximum occupation numbers $n_{\mathrm{occ}}^{\max }$. We also calculate the elastic conductance through a static neutral chain and a charged chain containing a static polaron in the center. One important result of our approach is that the inclusion of inelastic scattering strongly enhances the current in the tunneling regime compared to the case of elastic scattering. The transport becomes increasingly dominated by resonant tunneling through virtual polaron states as the injection energy approaches the top of the band gap. The first resonance in $\sigma(E)$ occurs at an injection energy very close to the charging energy $E_{\mathrm{ch}}$ of the isolated molecular chain (for $N_{\mathrm{a}}=40$, we calculate $E_{\mathrm{ch}}=0.53 \mathrm{eV}$ ). As expected, for

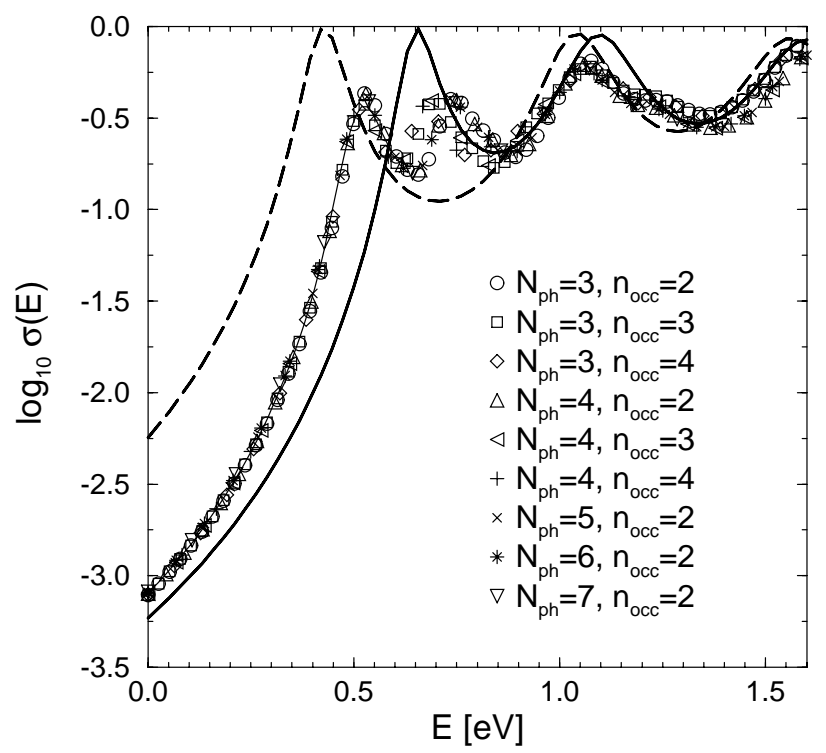

FIG. 2. Differential conductance $\sigma(E)$ (in units of $2 e^{2} / h$ ) through the wire $\left(N_{\mathrm{a}}=40\right) . \sigma(E)$ with inelastic scattering is represented by the symbols corresponding to different $N_{\mathrm{ph}}$ optical modes and $n_{\mathrm{occ}}^{\max }$. The thin solid line is a guide for the eye. The elastic conductance through the static neutral chain (solid line) and charged chain containing a static polaron (dashed line) is also shown ( $E=0$ is at mid-band-gap). any injection energy within the tunneling gap, the current decreases exponentially as the length of the wire increases (Fig. 3) for both elastic and inelastic calculations. The absolute value of $\sigma(E)$ is much larger when the $e$-ph coupling is included, and the apparent band gap (related to the slope of the curve) is smaller. Such effects become more important for longer molecular wires $\left(N_{\mathrm{a}} \geq 100\right)$. For shorter wires $\left(N_{\mathrm{a}} \leq 10\right)$, both calculations seem to converge; the tunneling process is too fast for the lattice to respond significantly to the presence of the electron, so the effects of $e$-ph coupling are less pronounced [25].

Finally, we present in Fig. 4 the quantum averaged induced atomic displacement $x_{j}^{[i]}$ of an atomic site $j$ when the tunneling electron is found on site $i$ [26]. These results are characteristic of phonon fluctuation-assisted tunneling via the dynamical formation of a virtual polaron inside the chain. The bond alternation, and hence the band gap, are locally suppressed around the tunneling electron. This distortion accompanies the electron through the chain, leaving a "wake" of distortion behind it. Such a persistent response of the lattice is not surprising in the absence of damping [22]. The displacements are slightly less than those for an isolated chain (not shown) because the lattice cannot respond fully to the tunneling electron. This effect manifests itself in the calculated conductance (Fig. 2); when tunneling, the current in the full quantum coherent calculations is less than the current that flows through a static chain where the polaron is "frozen in." This is because (a) the effective distortion in the quantum calculations is slightly smaller, and (b) the frozen-in polaron already exists so no elastic energy is needed to create it. Only fully quantum coherent calculations give qualitatively correct physics; it cannot be obtained from elastic scattering calculations through static chains (with or without disorder) or using a classical lattice.

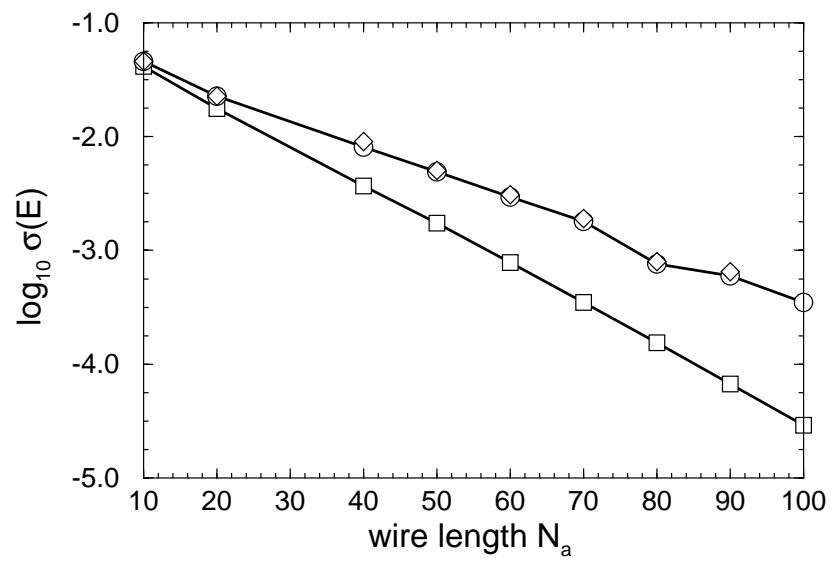

FIG. 3. Wire length dependence of $\sigma(E)$ (in units of $2 e^{2} / h$ ) for an injection energy $E=0.3 \mathrm{eV}$ inside the tunneling gap. The inelastic calculations are obtained for $N_{\mathrm{ph}}=4$ lowest optical modes, $n_{\mathrm{occ}}^{\max }=2$ (circles), and $n_{\mathrm{occ}}^{\max }=3$ (diamonds). The elastic conductance through the static neutral chain is also shown (squares). 


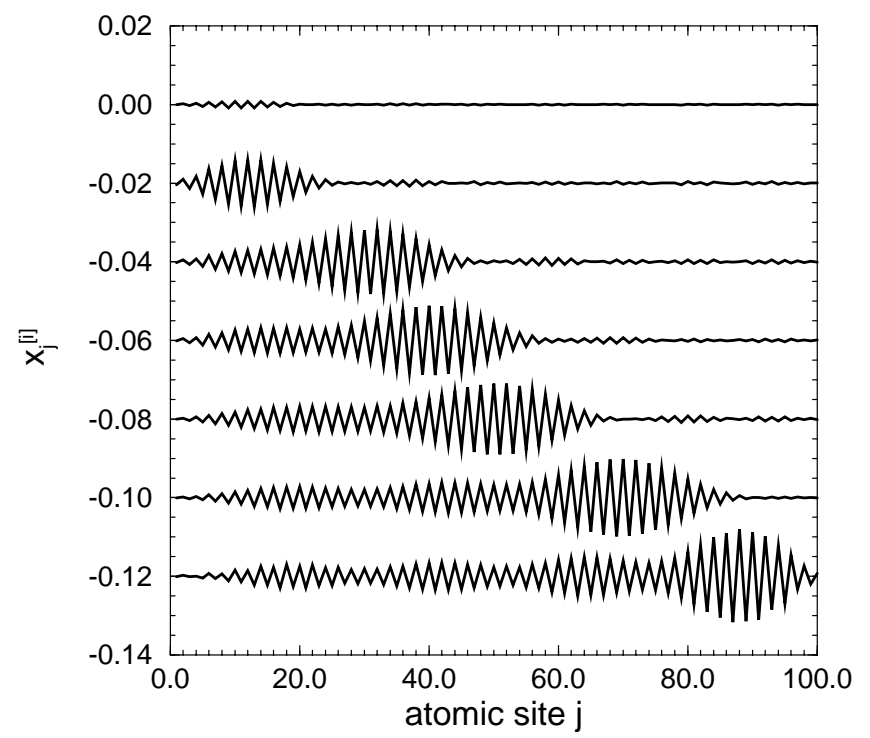

FIG. 4. Induced atomic displacements $x_{j}^{[i]}$ (in $\AA$ ) due to a tunneling electron (injection energy at midgap) for a $N_{\mathrm{a}}=100$ chain. The curves are shifted vertically (by 0.02 ) for clarity. The electron-projection $\hat{P}_{i}$ sites are $i=1,11,31,41,51,71$, and 91 for the top to the bottom curve, respectively. Calculations are performed for $N_{\mathrm{ph}}=8$ optical modes and $n_{\max }^{\text {occ }}=2$.

To conclude, we have presented the first calculation of inelastic effects on the conduction through molecular wires by explicitly considering realistic electron-phonon coupling. We have shown that the quantum coherent dynamical formation and transport of virtual polarons inside the molecular wire is crucial to describe the electronic transmission properties of such a system, and leads to a strongly increased current in the tunneling regime. This is the small-bias regime in which molecular wires will normally operate. Therefore, whenever tunneling conduction occurs in these or other one-dimensional atomic-scale wires [27] and most probably in conducting polymer wires [12], it will be by the mechanism identified here.

We thank A. M. Stoneham, L. Kantorovich, J. Gavartin, and A. Shluger for useful comments, and S. Shevlin for contributions to the present study. This work was supported by the U.K. EPSRC.

[1] S. J. Tans et al., Nature (London) 393, 49 (1998); S. Frank et al., Science 280, 1744 (1998).

[2] M. A. Reed et al., Science 278, 252 (1997).

[3] T. C. Shen et al., Science 268, 1590 (1995).

[4] T. Hitosugi et al., in Proceedings of the 3rd International Symposium on Advanced Physical Fields, Tsukuba-Japan, 1998 [J. Surf. Anal. 3, 312 (1998)]; T. Hitosugi et al., Phys. Rev. Lett. 82, 4034 (1999).

[5] L. Pizzagalli et al., Europhys. Lett. 38, 97 (1997).

[6] M. Magoga and C. Joachim, Phys. Rev. B 56, 4722 (1997); C. Joachim and J. F. Vinuesa, Europhys. Lett. 33, 635 (1996).
[7] N. D. Lang and Ph. Avouris, Phys. Rev. Lett. 81, 3515 (1998); N. D. Lang, Phys. Rev. B 55, 9364 (1997).

[8] M. Tsukada, N. Kobayashi, and M. Brandbyge, Prog. Surf. Sci. 59, 245 (1998).

[9] S. Datta et al., Phys. Rev. Lett. 79, 2530 (1997); M.P. Samanta et al., Phys. Rev. B 53, R7626 (1996).

[10] R. E. Peierls, Quantum Theory of Solids (Clarendon Press, Oxford, 1955), p. 110.

[11] A. J. Heeger et al., Rev. Mod. Phys. 60, 781 (1988).

[12] J. L. Bredas et al., Adv. Mater. 8, 447 (1996); I. H. Campbell et al., Phys. Rev. Lett. 76, 1900 (1996); E. M. Conwell and M.W. Wu, Appl. Phys. Lett. 70, 1867 (1997).

[13] M. Olson et al., J. Phys. Chem. B 102, 941 (1998).

[14] Z. G. Yu et al., J. Phys. Condens. Matter 11, L7 (1999).

[15] The parameters used are for trans-polyacetylene ( $t$-PA): the undimerized chain hopping integrals $t_{0}=2.5 \mathrm{eV}$, the electron-lattice coupling constant $\alpha=6.1 \mathrm{eV} / \AA$ and the spring constant $K=42 \mathrm{eV} / \AA^{2}$, taken from D.S. Wallace, Ph.D. thesis, University of Oxford, 1989.

[16] K. A. Chao and Y. Wang, J. Phys. C 18, L1127 (1985).

[17] The residence time can be estimated from the resonance widths in the transmission. We also calculated the Büttiker-Landauer tunneling time $\tau^{\mathrm{BL}}$ as in Ref. [23]. We find $\tau^{\mathrm{BL}} \approx 2$ to $4 \mathrm{fs}$ for $N_{\mathrm{a}}=40$ and $\tau^{\mathrm{BL}} \approx 8$ to $20 \mathrm{fs}$ for $N_{\mathrm{a}}=100$.

[18] For longer chains $\left(N_{\mathrm{a}} \approx 100\right.$, for example), the difference in the dimerization increases but is still in the range $15 \%-$ $17 \%$. This may be because the band gap decreases for longer chains. However, the atomic distortions due to charge injection are still well reproduced.

[19] These phonon energies are $\approx 0.1$ to $0.2 \mathrm{eV}$ for $N_{\mathrm{a}}=40$ to 100 , in agreement with experimental data for $t$-PA.

[20] The leads are 1D atomic chains with hopping integrals $\beta_{L, R}=3 \mathrm{eV}$. The matrix coupling elements between the end atoms of the chain and the first atoms of the left and right leads are $t_{L, R}=2.2 \mathrm{eV}$. The main results shown are not strongly dependent on those parameters.

[21] J. Bonča and S. A. Trugman, Phys. Rev. Lett. 75, 2566 (1995).

[22] J. Bonča and S. A. Trugman, Phys. Rev. Lett. 79, 4874 (1997).

[23] H. Ness and A.J. Fisher, J. Phys. Condens. Matter 10, 3697 (1998).

[24] The mean atomic displacement $\left\langle x_{i}\right\rangle$ is obtained from $\left\langle x_{i}\right\rangle=\sum_{q} V_{q}(i)\left\langle X_{q}\right\rangle$ where $\left\langle X_{q}\right\rangle$ is the mean displacement of the phonon mode $q: X_{q}=\sqrt{\hbar / 2 m \omega_{q}}\left(a_{q}^{\dagger}+a_{q}\right)$.

[25] Also, for short wires, the polaron displacement cannot be accommodated.

[26] The averaged atomic displacement $x_{j}^{[i]}$ of atom $j$ when the electron is found on site $i$ is obtained from $x_{j}^{[i]}=$ $\sum_{q} V_{q}(j) X_{q}(i)$ where $X_{q}(i)$ is the averaged displacement of mode $q$ when the electron is at site $i$, defined by $X_{q}(i)=\left\langle\hat{P}_{i} \hat{X}_{q} \hat{P}_{i}\right\rangle /\left\langle\hat{P}_{i}\right\rangle . \hat{P}_{i}$ is the electron wave function projector onto the atomic site $i$.

[27] Another system to which this mechanism will be relevant is the $\mathrm{DB}$ wire on the H-passivated $\mathrm{Si}(001)$ surface for which Peierls distortion occurs [4,28] and where a polaronlike defect is formed due to carrier injection [29].

[28] P. Doumergue et al., Phys. Rev. B 59, 15910 (1999).

[29] D. R. Bowler and A. J. Fisher (unpublished). 